\title{
A DESIGN TOOL FOR LIQUID ROCKET ENGINE INJECTORS
}

\author{
R. Farmer and G. Cheng, SECA, Inc., Huntsville, AL \\ H. Trinh and K. Tucker, NASA, Marshall space Flight Center, $\mathbf{L}$
}

\begin{abstract}
A practical design tool which emphasizes the analysis of flowfields near the injector face of liquid rocket engines has been developed and used to simulate preliminary configurations of NASA's Fastrac and vortex engines. This computational design tool is sufficiently detailed to predict the interactive effects of injector element impingement angles and points and the momenta of the individual orifice flows and the combusting flow which results. In order to simulate a significant number of individual orifices, a homogeneous computational fluid dynamics model was developed. To describe sub- and supercritical liquid and vapor flows, the model utilized thermal and caloric equations of state which were valid over a wide range of pressures and temperatures. The model was constructed such that the local quality of the flow was determined directly. Since both the Fastrac and vortex engines utilize RP-1/LOX propellants, a simplified hydrocarbon combustion model was devised in order to accomplish three-dimensional, multiphase flow simulations. Such a model does not identify drops or their distribution, but it does allow the recirculating flow along the injector face and into the acoustic cavity and the film coolant flow to be accurately predicted.
\end{abstract}

\section{Introduction}

To provide detailed design information on liquid rocket engine injector performance, a computational simulation which relates the configuration of the injector faceplate to the flowfield generated in the engine is required. Injector elements are generally designed to form overlapping sprays to mix the fuel and oxidizer streams as they enter the combustor. Most engines operate at conditions such that the sprays are supercritical, or soon become so as they enter the combustor. Historically, patternator cold-flow experiments are used to estimate inlet mixture ratio distributions, ${ }^{1,2}$ and the JANNAF standard axisymmetric finite-rate (TDK) computer code ${ }^{3}$ is used to predict performance. Computational fluid dynamics (CFD) codes, like the EDNS-RFV code used herein, can be used to replace the TDK code without compromising the results. If the TDK code is used, the initial mixture ratio distributions must be estimated. The FDNS-RFV CFD code calculates these distributions directly. Even when using the CFD codes, three-dimensional effects are seldom simulated. Despite the major effort that has been devoted to analyzing spray effects by tracking drops, such analyses are too computationally intensive to describe the flows from the complex injector configurations in real engines. In addition, the underlying physics of stripping and atomizing supercritical liquid drops, where the surface tension does not exist, is not well understood. This work approximates the full spray dynamics by using a homogeneous spray model which retains the liquid-vapor flow features by using a real fluid thermodynamics model that describes the local quality of the fluid. Such an approximation is essential in order to simulate many of the important three-dimensional aspects of the combustor flow. The attributes of this model and examples of its use are presented herein.

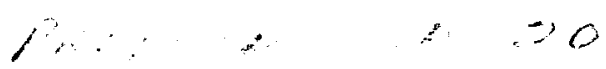




\section{Methodology}

To describe sub- and supercritical spray combustion with a homogeneous model, multiphase, multicomponent, reacting turbulent flows must be modeled. The methods of representing these effects are described as follows.

Thermal and caloric equations of state, vapor pressure, heat of vaporization, surface tension, and transport properties are modeled with the equations of state proposed by Hirshfelder, et $a^{5,6}$ (we term these the HBMS equations of state) and with conventional correlations, ${ }^{7}$ for the other properties. The property correlations used were not chosen for their absolute accuracy, but for their validity over a wide range of temperatures and pressures and for requiring a minimum of data to describe a particular species. These correlations are explicit in density and temperature.

HBMS thermal equation of state:

$$
\frac{P}{P_{c}}=\sum_{j=1}^{4} T_{r}^{j-2} \sum_{i=1}^{6} B_{i j} \rho_{r}^{i-2} ; T_{r}=\frac{T}{T_{c}} ; \rho_{r}=\frac{\rho}{\rho_{c}}
$$

HBMS caloric equation of state:

$$
\frac{H-H_{0}}{R T}=Z_{c} \int_{f}^{\rho_{r}}\left[\frac{P}{T_{r}}-\left(\frac{\partial P}{\partial T_{r}}\right]_{\rho_{r}}\right] \rho_{r}^{-2} d \rho_{r}+Z_{c} \frac{P}{\rho_{r} T_{r}}-1
$$

These equations are based on the "theorem of corresponding states" for real fluids, which essentially means that the $\mathrm{p}-\mathrm{v}-\mathrm{T}$ relations for all species are similar if these variables are normalized with their values at the critical point,i.e. if reduced values are used. The reduced values in these equations are indicated with a subscript $r . H_{o}$ is the ideal gas species enthalpy. $\mathrm{Z}_{\mathrm{c}}$ is the compressibility for a given species at the critical point. The HBMS equations are attractive to use because arbitrary correlations for vapor pressure, heat of vaporization, and liquid densities can be used. Since multi-component fluid/vapor mixtures may be present in the flowfield, the mixture properties are calculated by the additive volume method. This means that multiphase mixtures are treated as ideal solutions. To simulate RP-1, a surrogate fuel composed of several representative species was postulated. Properties of these pure species were then estimated and a mixture formed ${ }^{8}$. When not otherwise available, properties were estimated by using bond energies for their molecular structure with Benson's methodology'. For conditions where the species become ideal gases, the thermodynamic data from the CEC code ${ }^{10}$ or from the Thermodynamic Research Center at Texas A\& $\mathbf{M}^{11}$ were used.

The CFD solver used was the Finite-Difference Navier-Stokes code with provision for using real fluid properties, the FDNS-RFV code. This code is pressure based; it differs from an ideal gas code in the methodology used to relate the pressure correction to the continuity equation and of course in the properties subroutines used. The pressure correction $\left(p^{\prime}\right)$ equation used in the FDNS-RFV code is: 


$$
\begin{gathered}
\frac{\beta_{p} p^{\prime}}{\Delta T} \nabla \cdot\left(u_{i} \beta_{p} p^{\prime}\right)-\nabla \cdot\left(\rho^{*} D_{p} \nabla p^{\prime}\right)=-\nabla \cdot\left(\rho^{*} u_{i}\right)-\frac{\rho^{*}-\rho^{n}}{\Delta t} \\
p^{n+1}=p^{n}+p^{\prime} ; \quad \beta_{p}=\gamma / a^{2} ; \quad u_{i} \approx-D_{p} \nabla p^{\prime}
\end{gathered}
$$

where the superscripts * and $\mathrm{n}$ denote the value at the intermediate and previous time steps, respectively. $D_{p}$ is the inverse of the matrix of the coefficients of the convective terms in the finite-difference form of the inviscid equations of motion. This is not an obvious definition, but is one which has made the FDNS-RFV code a useful solver. The sound speed used in the pressure correction equation is that calculated for the real fluid multi-component mixture.

To describe both the Fastrac and vortex engines, a simplified RP-1/LOX combustion model was devised in order to accomplish three-dimensional, multiphase flow simulations. Global RP-1 pyrolysis, partial oxidation, and soot formation steps were used, and the combustion was completed with a wet $\mathrm{CO}$ elementary reaction mechanism. Hence, in addition to the hydrocarbons, the species $\mathrm{CO}, \mathrm{CO}_{2}, \mathrm{H}_{2} \mathrm{O}, \mathrm{H}, \mathrm{O}, \mathrm{OH}$ were considered. Using the wet $\mathrm{CO}$ mechanism allows for disassociation in the chamber and recombination in the nozzle, which is necessary to obtain accurate temperatures in the simulation. Averaged data from gas generator tests ${ }^{12}$ and from subscale motor test ${ }^{13}$ were used to establish mean molecular weights, temperature, and soot levels for which the combustion model was tuned. The global submodel used for LOX/RP-1 combustion is shown in Table 1. The RP-1 is allowed to pyrolyze to $\mathrm{C}_{2} \mathrm{H}_{4}$ surrogate (not ethylene) which has the heat capacity of ethylene and the heat of formation of RP1. Thus, the pyrolysis is thermally neutral, and the heat of combustion matches that of RP- 1 . The $\mathrm{C}_{2} \mathrm{H}_{4}$ surrogate is used as the soot precursor. Soot is assumed to be $\mathrm{C}_{96} \mathrm{H}_{24}$, circumcircumcoronene, in order to treat it as a gas with a density like a solid. This model was designed to allow easier tuning of the reactions to match observed combustion product molecular weight and flame temperatures. The molecular weight and flame temperatures of a low mixture ratio used for validation were those observed by Aerojet in gas generator simulation experiments. Matching these properties also matches the sound speed of the combusting mixture. Soot oxidation was neglected in the simulations reported herein, because there was insufficient oxygen for the soot oxidation to occur since the global $\mathrm{C}_{2} \mathrm{H}_{4}$ oxidation reaction was faster than the expected soot oxidation rate. A finite-rate vaporization model, such as that suggested by Johnson, et $\mathrm{al}^{13}$ could be used to account for the heat and mass transfer effects between the RP-1 liquid drops and the surrounding hot gases, if sufficient data were available to justify its use.

In all cases simulated, a $\mathrm{k}-\epsilon$ turbulence model was used to close the mass averaged transport equations solved by the code. Our experience is that this incompressible turbulence model overestimates the mixing in a combusting flowfield. However, since the liquid propellants are also mixed by this model, we concluded that there are currently insufficient data to better tune the turbulence model. Since a very fine grid is used to describe the details of the individual injector elements, the turbulence model essentially describes the subgrid turbulence 
Table 1. Combustion Kinetic Models for RP-1/ $\mathbf{O}_{2}$

$$
\begin{aligned}
& \mathrm{RP}-1 \rightarrow 6.2 \mathrm{C}_{2} \mathrm{H}_{4} \\
& \text { Rate }=\mathrm{AT}^{\mathrm{B}} \exp \{-\mathrm{E} / \mathrm{RT}\}[\mathrm{RP}-1]^{0.5} \\
& \mathrm{~A}=3.0117 \times 10^{10}, \mathrm{~B}=0, \mathrm{E} / \mathrm{R}=2.523 \times 10^{4} \\
& \mathrm{C}_{2} \mathrm{H}_{4}+\mathrm{O}_{2} \rightarrow 2 \mathrm{CO}+2 \mathrm{H}_{2} \\
& \text { Rate }=\mathrm{AT}^{\mathrm{B}} \exp \{-\mathrm{E} / \mathrm{RT}\}\left[\mathrm{C}_{2} \mathrm{H}_{4}\right]^{0.5}\left[\mathrm{O}_{2}\right]^{1.0} \\
& \mathrm{~A}=1.29 \times 10^{15}, \mathrm{~B}=1, \mathrm{E} / \mathrm{R}=2.516 \times 10^{4}
\end{aligned}
$$

$48 \mathrm{C}_{2} \mathrm{H}_{4} \rightarrow$ Soot $+84 \mathrm{H}_{2}$

$$
\begin{aligned}
& \text { Rate } \left.=\mathrm{AT}^{\mathrm{B}} \exp \{-\mathrm{E} / \mathrm{RT}\} \mathrm{C}_{2} \mathrm{H}_{4}\right]^{2.0}\left[\mathrm{O}_{2}+\epsilon\right]^{-0.5} \\
& \mathrm{~A}=5.1308 \times 10^{12}, \mathrm{~B}=-2, \mathrm{E} / \mathrm{R}=1.611 \times 10^{4}, \epsilon=0.001 \rho / \mathrm{M}_{\mathrm{O}_{2}} \mathrm{~g}-\mathrm{mole} / \mathrm{cm}^{3}
\end{aligned}
$$

effects. Such an analysis is a form of large scale eddy simulation, the large scale eddies being roughly of the size of the near flowfield of a single injector element. The homogeneous spray model was used to simulate a single element like-on-like (LOL) impinger injector element and a single element unlike impinger element for the configuration and flow conditions used in the cold-flow test in the JANNAF data base. Results for the LOL case are shown in Figure 1, as well as the cold flow correlations. The mass flux distributions look very similar one-inch from the faceplate, and further downstream the correlations predict faster decay. The comparisons of mixture ratio distribution are shown in Figure 2. Since all of the injected flow was not recovered in the cold flow experiments and since the non-uniformity of the CFD predicted mixture ratio distributions appear more realistic, the CFD simulations appear to give as useful a result as the cold-flow data correlations. In all cases, regions of low mass flux should be considered immaterial. Furthermore, the cold-flow data are for a very limited range of configurations and flowrates. The CFD simulations could be used to parametrically and systematically evaluate the effects of impingement points, impingement angles, and injection velocities. Unless significant improvements are made in conducting the cold-flow experiments, such detailed information cannot be obtained.

It could be argued that the homogeneous model does not accurately account for the mixing due to the interaction of drop clouds and that the liquid and vapor streams are not in thermal equilibrium. However, efforts to measure drop distributions in cold-flow LOL sprays ${ }^{14}$ have been unsuccessful because of the very dense drop concentrations in the spray proper. No spray combustion data exist with which to evaluate a model for the severe conditions which exist in a rocket motor. For typical rocket engines, the chamber conditions are mostly supercritical; thus, the surface tension of the liquid is reduced to zero and the heat transfer and drag coefficients between the drops and the surrounding gases becomes ill defined. For example, for LOX/RP-1 rocket engines, the chamber pressure is typically well above the critical pressure of RP-1, and for LOX the temperature quickly exceeds that of oxygen (frequently the pressure is also supercritical). Under these conditions the drops which do exist are far more unstable than those which are seen in cold-flow experiments. ${ }^{15}$ Experiments conducted by the Air Force Research Laboratory, ${ }^{16}$ show that supercritical injection of a round jet of liquid nitrogen into gaseous nitrogen behaves like a gas jet. This is precisely the behavior predicted by the homogeneous spray model. If viable data to describe droplet phenomena in high-pressure combusting flows do become available, the homogeneous spray model has two parameters (the 
finite vaporization rate and the inter-element mixing rate) which may be adjusted to match such data. These changes would add negligible complexity to the CFD simulation.

\section{$\underline{\text { Numerical Simulations }}$}

Two examples of the use of the homogeneous spray code will be presented: the Fastrac and the vortex motor. These engines were chosen because at the time this work was done they were under development by NASA/MSFC. The simulations were made for preliminary engine configurations to give insight as to how the injectors were expected to operate. The analyses presented do not represent the best and final design on either of these engines.

\section{The Fastrac Engine}

A segment of the Fastrac main combustion chamber near the corner of the faceplate and the acoustic cavity was analyzed. This segment simulates several like-on-like injector elements of the Rev.-C configuration and was chosen to evaluate the temperatures in the acoustic cavity and along the chamber wall. The number of elements were limited so that the simulation could be efficiently conducted on a PC. One injector element on row \#8 and one element on row \#7 were simulated. The number of film coolant holes was arbitrarily increased by $6 \%$ to make the computational domain symmetric in the circumferential direction. The first 2.5 inches downstream of the faceplate were simulated. About $110 \mathrm{~K}$ grid points were used for this simulation. This amounts to about 25 grid points per orifice opening. The boundary conditions and injector geometry and flow rates (per hole) are presented in Table 2.

Table 2. The Inlet Flow Conditions and Configurations of the Fastrac Injector Rev.-C)

\begin{tabular}{||l|c|c|c|c|c||}
\hline \multirow{2}{*}{} & \multicolumn{2}{|c|}{ Row \#7 } & \multicolumn{2}{c|}{ Row \#8 } & Coolant \\
\cline { 2 - 6 } & Oxidizer & Fuel & Oxidizer & Fuel & \\
\hline Pressure (psi) & 633 & 633 & 633 & 633 & 633 \\
\hline Temperature ( $\left.{ }^{\circ} \mathrm{R}\right)$ & 190 & 540 & 190 & 540 & 540 \\
\hline Density (lbm/ft ${ }^{3}$ & 67.14 & 46.44 & 67.14 & 46.44 & 46.44 \\
\hline Diameter (inch) & 0.105 & 0.069 & 0.105 & 0.069 & 0.042 \\
\hline Mass flow rate (lbm/s) & 0.3839 & 0.1381 & 0.3965 & 0.1381 & 0.0625 \\
\hline Impinging angle & $40^{\circ}$ & $50^{\circ}$ & $40^{\circ}$ & $50^{\circ}$ & $45^{\circ}$ swirl \\
\hline Cant angle & $8^{\circ}$ & $-25^{\circ}$ & $-16.5^{\circ}$ & $-25^{\circ}$ & $18^{\circ}$ \\
\hline
\end{tabular}

The predicted velocity and temperature fields are shown in Figure 3 . The mean temperature in the cavity is $2000^{\circ} \mathrm{R}$ which is in good agreement with measured values. The isotherms are essentially the mixture ratios in this region. Notice the numerous small eddies and 
recirculation zones which predicted by the analysis; these constitute the "large scale" eddies which are a result of the numerical solution of the transport equations. As the liquid propellants jet into the chamber the streams collide and $\mathrm{mix}$. The combustion occurs where the propellants have intermingled enough to form a combustible mixture. As the combustion occurs, the hot gases expand and offer additional resistance to forming a well mixed flow. The simulation indicates extreme unmixedness, which is probably the case near the faceplate. Note that the cross-winds near the injector face are predicted, and these velocities create the flow which is induced into the acoustic cavity. The solution procedure was initialized by a cold flow calculation performed by not allowing the flow to react. The flame initiation was accomplished by assigning a hot spot (a few grid points were set to $1500^{\circ} \mathrm{K}$ ) near the wall. The major advantage offered by this simulation is that the injected propellants enter at the correct velocity, momentum, and energy levels for the engine. (Therefore, the predicted temperatures, mixture ratios, and velocities should be realistic.) The downstream boundary conditions are determined by mass conservation. The side of the control volume adjacent to the flows from the elements closer to the centerline are treated with slip boundary conditions. The sides of the control volume bounding the circumferential direction are treated with periodic boundary conditions in order to account for the swirl introduced by the film cooling flow. Ideally, a pie-shaped section of the motor would be analyzed, but the configuration of the injector face was symmetric only in a $90^{\circ}$ quadrant. Also, too many rows of elements were used for all of them to be simulated on a PC. Nevertheless, the computational domain used was sufficient to allow a valid simulation of the flow through the acoustic cavity and near the chamber wall.

\section{The Vortex Engine}

The concept of a vortex engine is to use the vorticity of the injected propellants to provide longer residence times in the chamber and, hopefully, better mixing. A preliminary design of such a vortex motor was analyzed. This motor introduced the propellants through 16 pairs of unlike injector elements, tangential to and around the circumference of the combustion chamber. The injected propellants therefore produced swirl in the chamber to enhance the residence time in the short chamber. The propellants are injected at a high enough velocity to create the swirl, and they do mix very well at the injection point. In a conventional motor, the many injector elements provide well mixed propellants, whereas the vortex motor depends on passive mixing of the propellant streams. A one-sixteenth pie-shaped section of the chamber was simulated. Periodic boundary conditions were used on the sides of the section. The simulation was conducted only part way down the expansion nozzle, but this was far enough to evaluate the mixing efficiency in the chamber. Operating conditions for this engine are expected to be a mixture ratio of $2.6,4.15 \mathrm{lb} / \mathrm{sec}$ total propellant flow, 1000 psia chamber pressure.

The chamber diameter is $2.35 \mathrm{in}$, throat diameter 0.96 in, and exit diameter of 3.03 in for an expansion ratio of 9.96 . The diameters of the RP-1 injector and the LOX injector are 0.04 and 0.06 in, respectively. The impingement angle is $36 \mathrm{E}$ between the unlike injector elements. The computational domain consists of $90 \mathrm{~K}$ grid points.

The predicted temperature field and species concentrations are shown in Figure 4. The mixture ratio contours would correspond exactly to the isotherms. Hence, the propellants remain largely unmixed for these flow conditions. Streamlines from the fuel side of the injector element (i.e. the orifices nearer to the faceplate), the oxidizer side of the injector element, and from a near-wall point located in between the fuel and oxidizer elements are shown in Figure 5. The 
simulation shows the flow to be very unmixed and to possess moderate swirl. The turbulence mixing was simulated with the incompressible $\mathrm{k}-\epsilon$ model, which is believed to overestimate the mixing. However, even with the somewhat longer residence time due to the swirl, efficient mixing is not accomplished with this design. The predicted thrust from the CFD solution is $80 \%$ of that obtained from a one-dimensional, fully mixed, equilibrium solution. The predicted mean chamber pressure was 940 psia, with an 80 psia pressure difference between the injector wall and the engine centerline. NASA is building and testing this preliminary design as a baseline for the vortex concept, so a final verification of the CFD predictions will be made.

\section{$\underline{\text { Closure }}$}

A homogeneous spray combustion model for analyzing liquid rocket engines has been developed. The unique feature of the CFD model is that real configurations of liquid rocket engines can be simulated from the individual orifices in the injector all the way to the nozzle exit. Rational approximations are made to the spray dynamics involved to provide this engineering CFD model. Real fluid properties are used to represent the liquid, vapor, and twophase flow regions in the engine. For these multi-component combusting flows, some of the components may be subcritical while others are supercritical. Examples of this methodology are given for a segment of the Fastrac combustion chamber and for a preliminary design of a vortex motor, both of which utilize LOX/RP-1 propellants. The examples shown were prepared on a single Pentium II, $450 \mathrm{mHz}$ processor. Depending on the computer used a larger number of injector elements (or fraction of the engine) could be analyzed. However, even with the fulltime use of a Cray a solution of an entire Fastrac engine is not yet possible. However, a complete analysis of the vortex engine with its 32 injector elements which are of good circumferential symmetry is now possible on a PC. The code reported herein is currently being modified to use parallel processing to expedite the analysis of more complex liquid rocket engines.

\section{$\underline{\text { References }}$}

1. Combs, L.P., "Catalog of Injector Spray Correlations," Rpt. on NAS7-746, Rocketdyne, a Div. of North American Rockwell Corp., Canoga Park, CA, June 1972.

2. Pieper, J.L., "Single Element Cold Flow Test Report," Rpt. on F04611-85-C-0100, Aerojet TechSystems Co., Sacramento, CA, Nov. 1988.

3. Nickerson, G.R., et al, "Two-Dimensional Kinetics (TDK) Nozzle Performance Computer Program," Vols. I-III, Rpt. No. SN91, Software and Engineering Associates, Inc., Mar. 1989.

4. Cheng, G.C., "Programmer's and User's Manual for the FDNS-RFV CFD Code," SECA, Inc., Huntsville, AL, May 1998.

5. Hirschfelder, J.O., et al, "Generalized Equation of State for Gases and Liquids," I\&EC, $\underline{50}$, pp. 375-385, 1958.

6. Hirschfelder, J.O., et al, "Generalized Excess Functions for Gases and Liquids," I\&EC, $\underline{50}$, pp.386-390, 1958. 
7. Reid, R.C., et al, The Properties of Gases \& Liquids, 4th ed., McGraw-Hill, 1987.

8. Farmer, R.C., et al, "Propulsion Chemistry for CFD Applications," SECA-FR-97-08, SECA, Inc., Huntsville, AL, 1997.

9. Benson, S.W., Thermochemical Kinetics, 2nd Ed., Wiley \& Sons, NY, 1976.

10. Gordon, S. and B.J. McBride, "Computer Program for Calculation of Complex Chemical Equilibrium Compositions, Rocket Performance, Incident and Reflected Shocks, and ChapmanJouget Detonations," NASA SP-273, 1971.

11. Wilhoit, R.C., "TRC Current Data News," 3, No. 2, Thermodynamics Research Center, Texas A\&M University, 1975.

12. Hernandez, R., "Carbon Deposition Model for Oxygen-Hydrocarbon Combustion," RPT/AA0631-a/087, Aerojet TechSystems Co., Sacramento, CA, Sep. 1987.

13. Johnson, C., et al, "Prediction of Soot Production in Kerosene Fueled Liquid Rocket Engines," JANNAF 35th Combustion Subcommittee, Tucson, AZ, 1998.

14. Hautman, D.J., "Spray Characterization of Like-On-Like Doublet Impinging Rocket Injectors," AIAA 91-0687, 29th Aerospace Sciences Meeting, Jan. 1991.

15. Pike, R.W., et al, "Single Droplet Combustion of RP-1 Rocket Fuel," Paper 112t, AIChE 1998 Annual Meeting, Miami Beach, FL, Nov. 1998.

16. Chehroudi, B., et al, "Initial Growth Rate and Visual Characteristics of a Round Jet into a Sub- to Supercritical Environment of Relevance to Rocket, Gas Turbine, and Diesel Engines," AIAA 99-0206, 37th Aerospace Sciences Meeting, Reno, NV, Jan. 1999. 


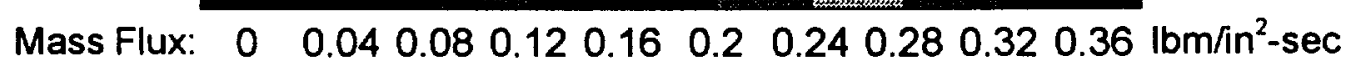

(a) $Z=1$ inch

(b) $Z=1.5$ inch

(c) $Z=2$ inch
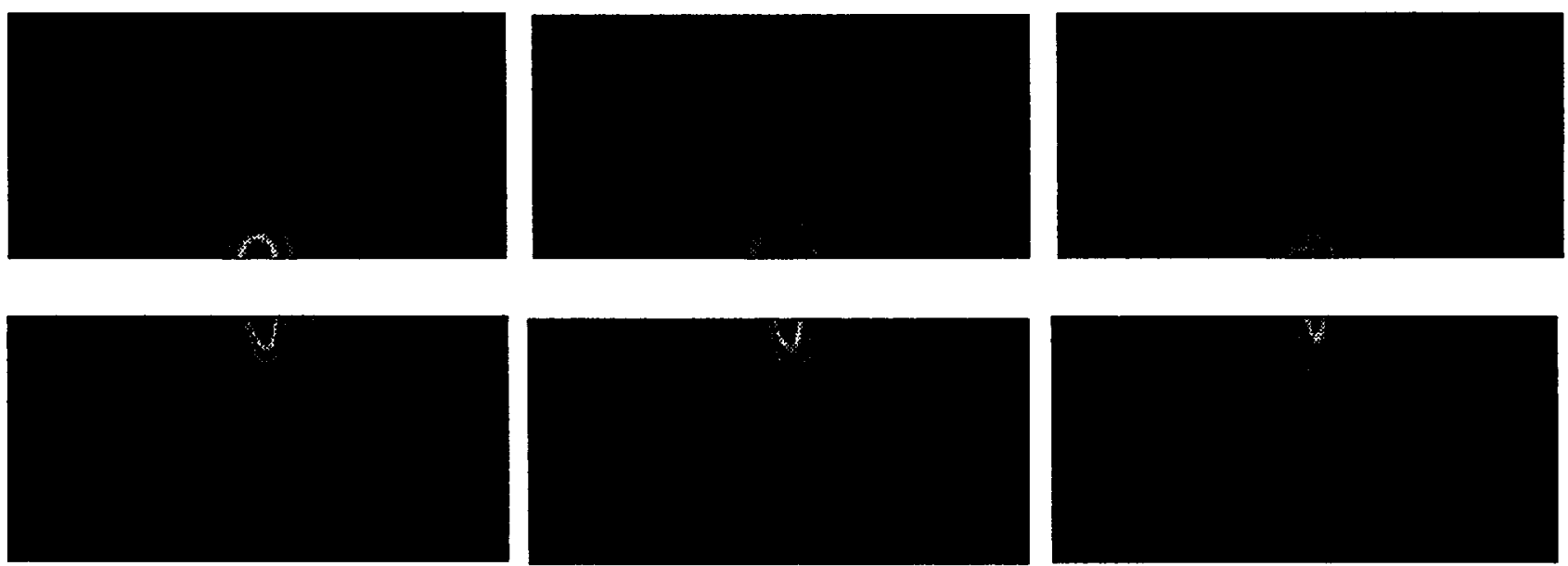

Top: Empirical Solution; Bottom: CFD Solution

Figure 1 Mass Flux Distributions for Like-on-Like Impinger

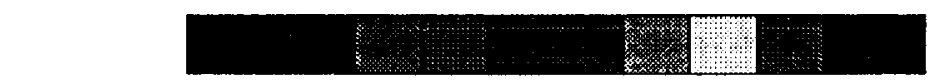

O/F Ratio: 11.21 .41 .61 .8222 .22 .42 .62 .83

(a) $Z=1$ inch
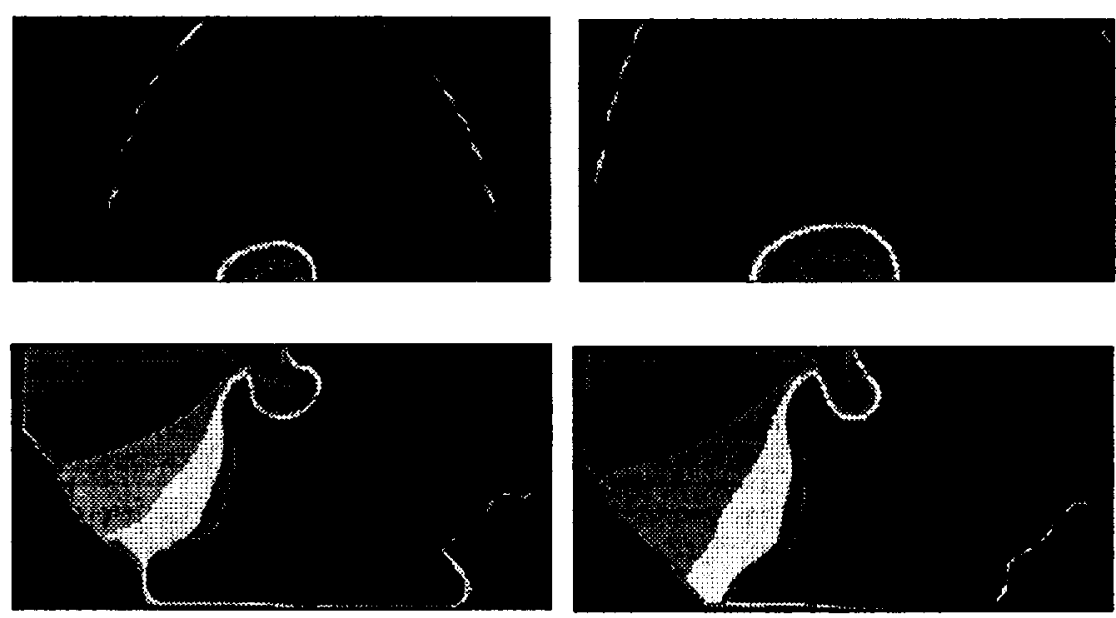

(b) $Z=1.5$ inch

(c) $Z=2$ inch
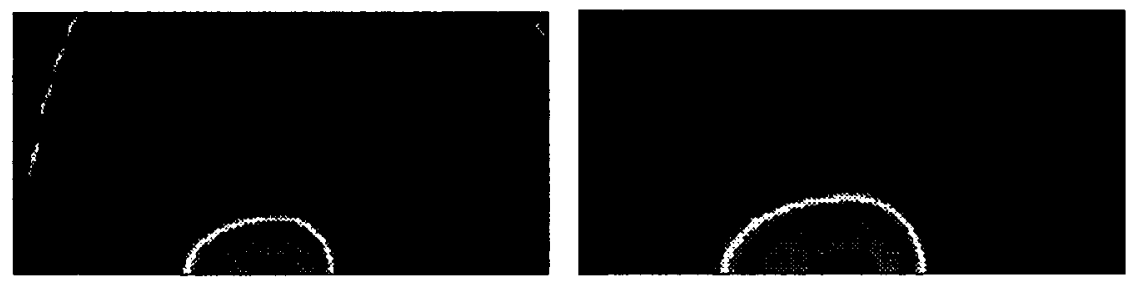

Top: Empirical Solution; Bottom: CFD Solution

Figure 2 O/F Ratio Distributions for Like-on-Like Impinger 


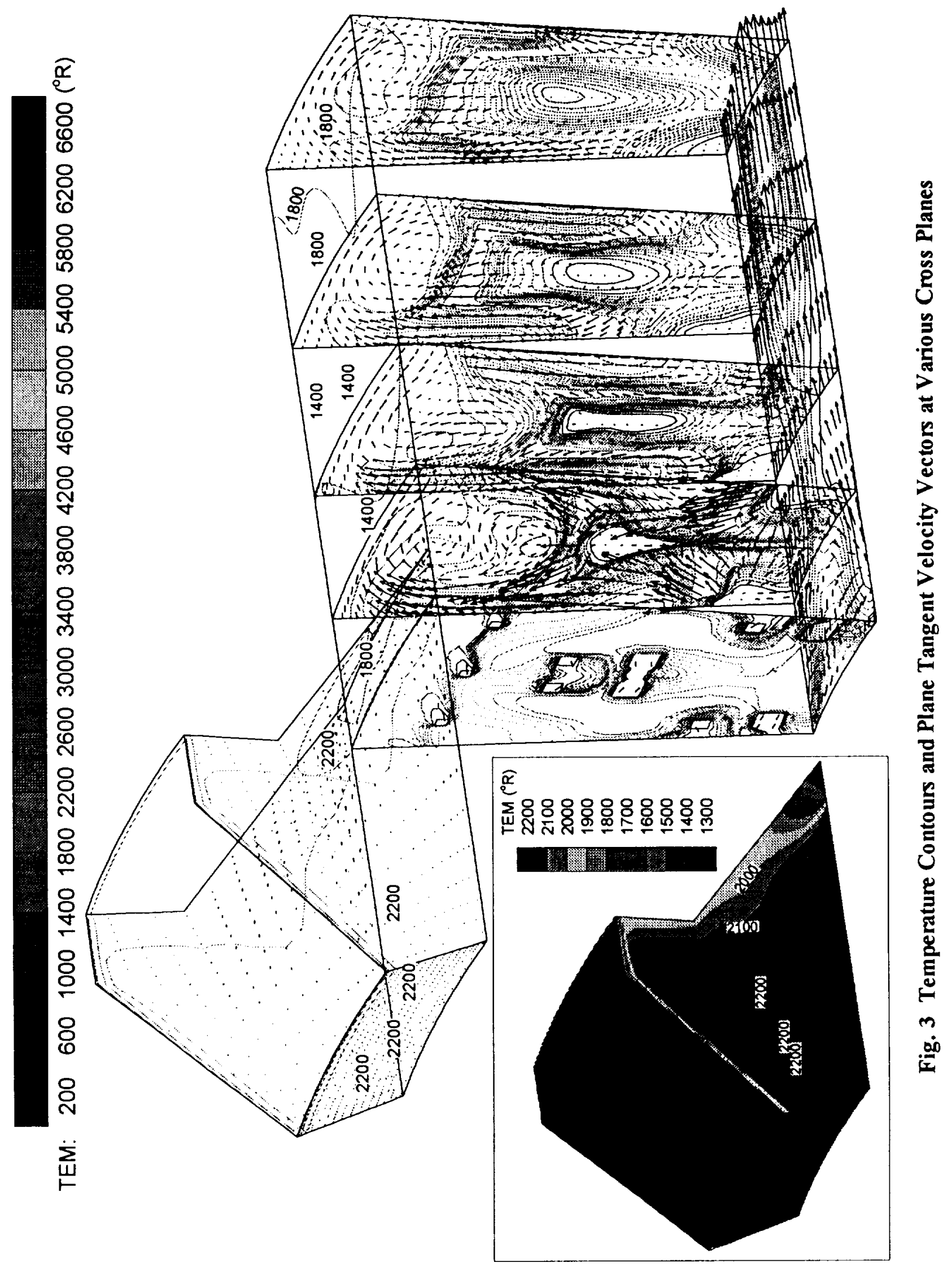



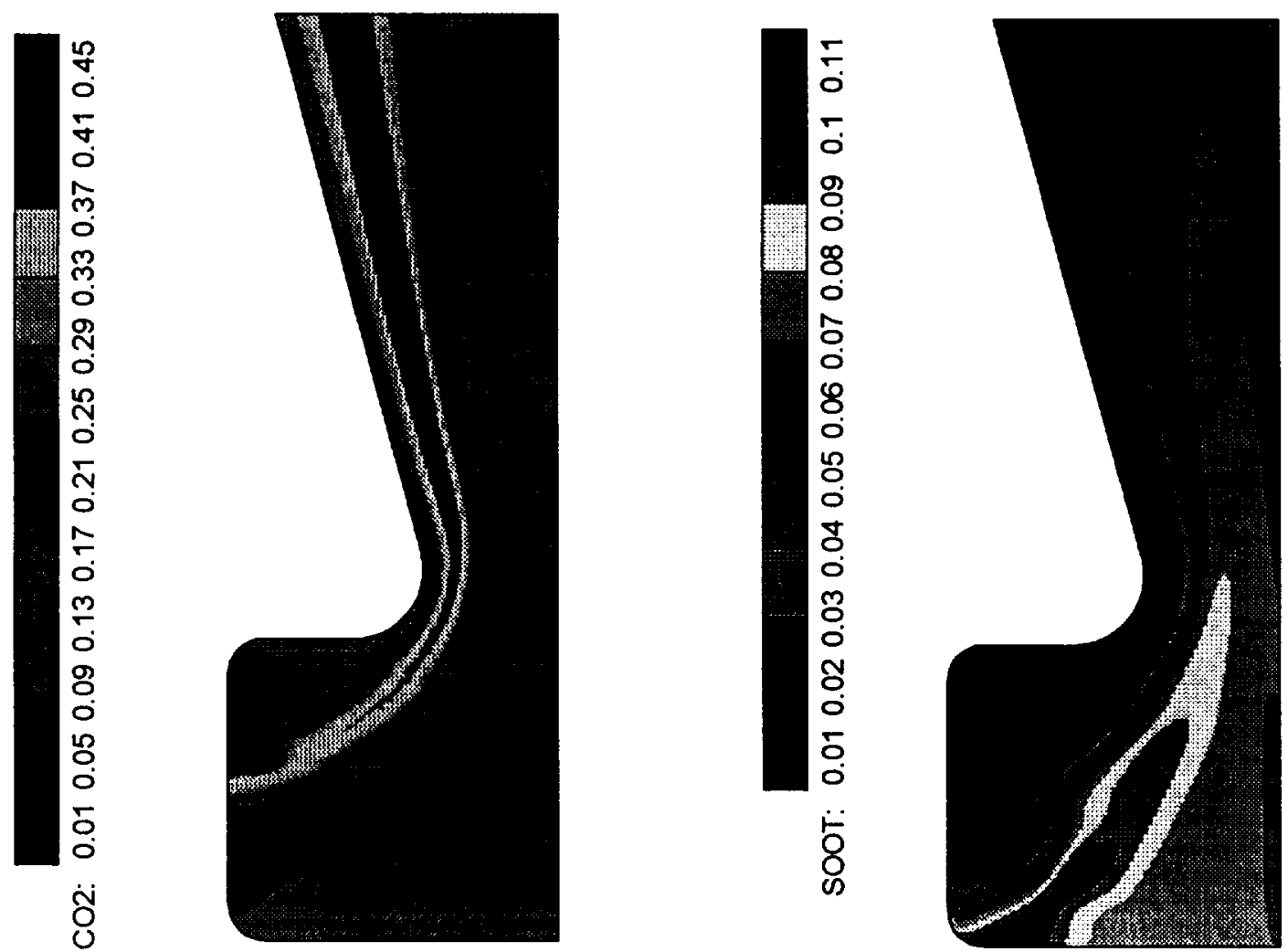

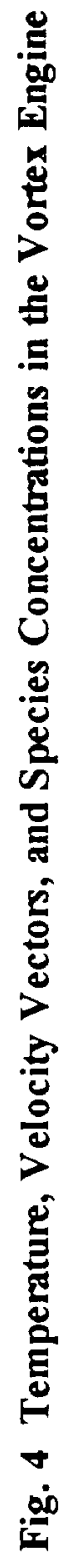
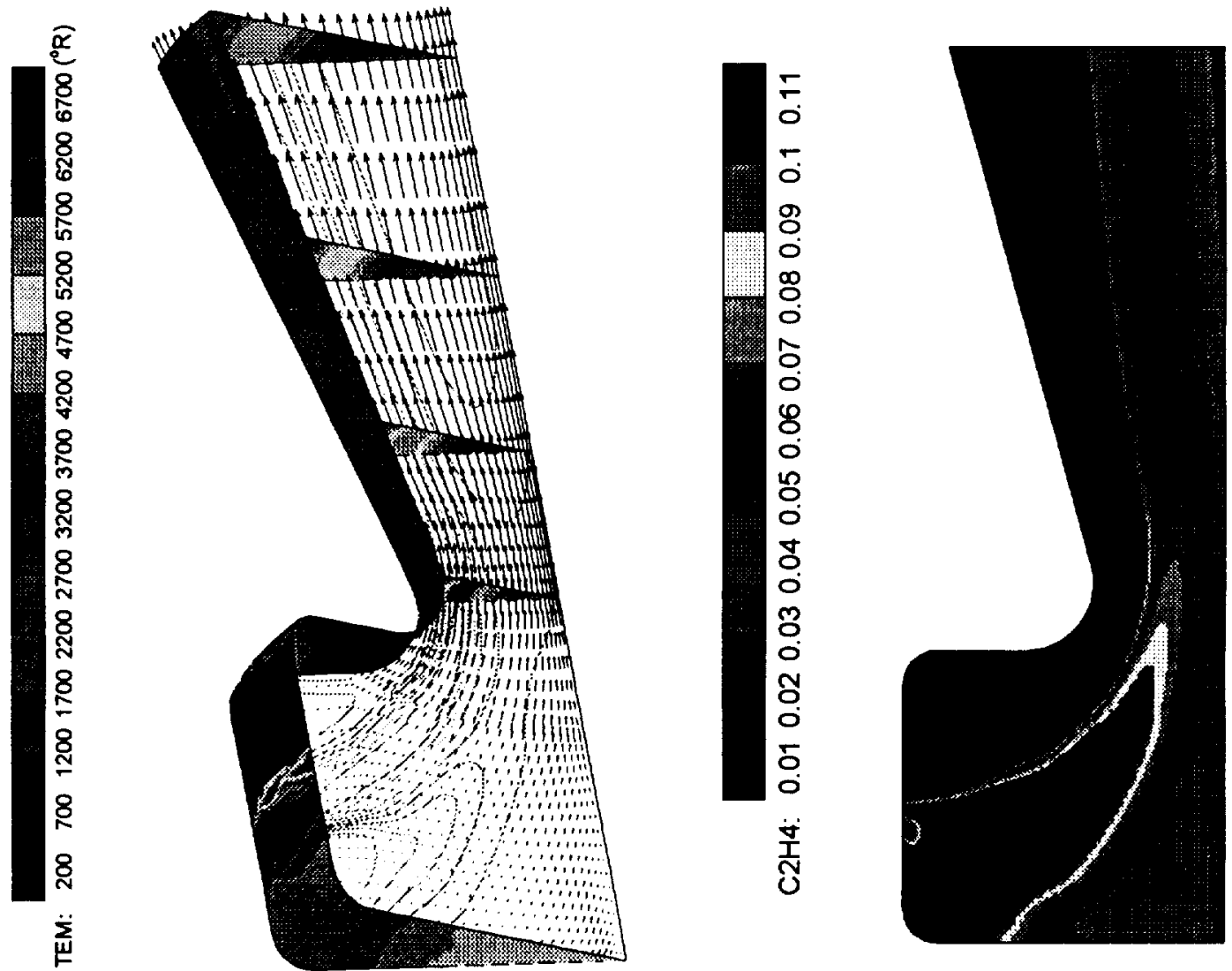
TEM: 200700120017002200270032003700420047005200570062006700 ('R)

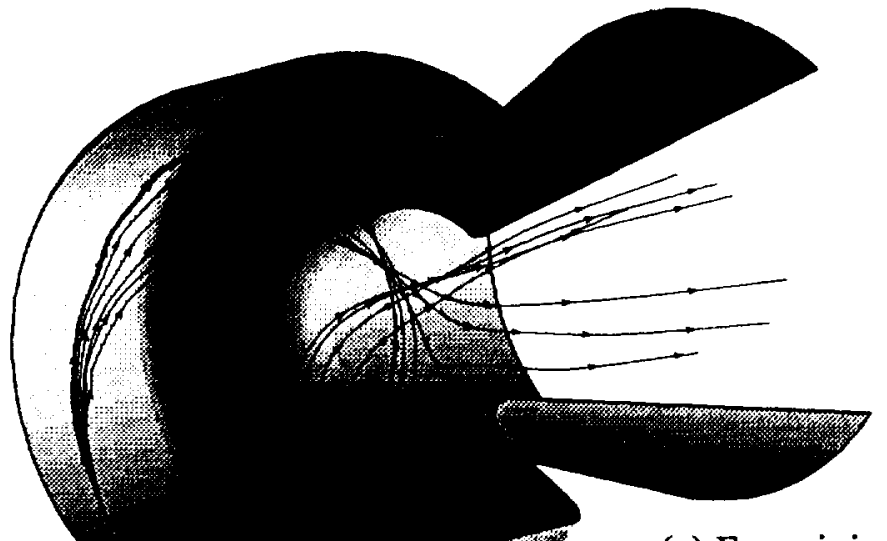

(a) From injected fuel hole
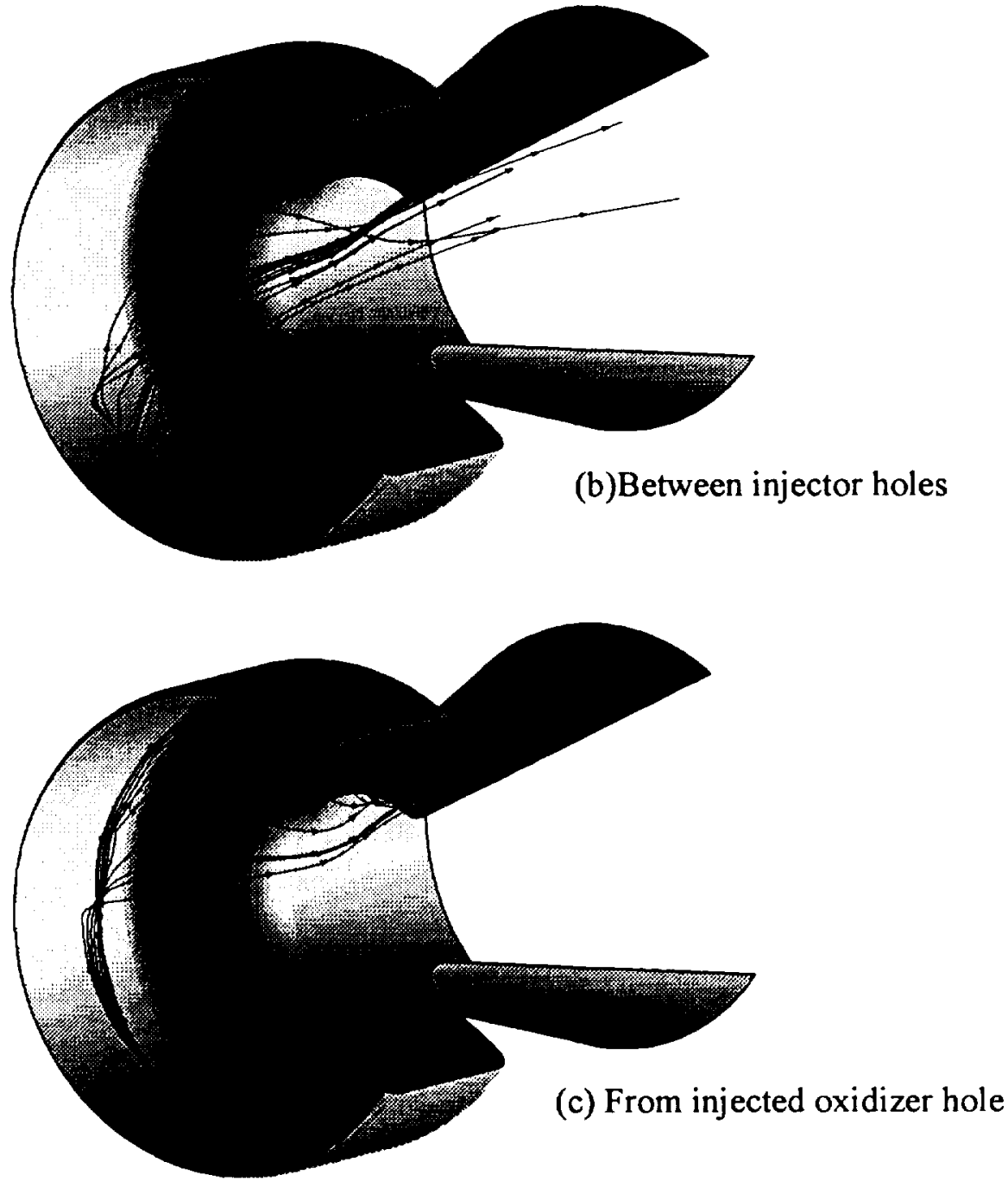

Figure 5 Selected Streamlines of the Vortex Engine 